Review Article

\title{
Indian Experimental Physical Chemists
}

\author{
HIRENDRA N GHOSH ${ }^{*}, 1,2$ \\ ${ }^{1}$ Institute of Nano Science and Technology, Mohali, Punjab 160 062, India \\ ${ }^{2}$ Radiation and Photochemistry Division, Bhabha Atomic Research Centre, Mumbai 400 085, India
}

(Received on 03 March 2019; Revised on 25 May 2019; Accepted on 05 June 2019)

\begin{abstract}
Although a lots of high-quality research work are going on in different area in the field of Chemical Sciences as a whole in several research laboratories (both in Institutes and Universities) in India, however, the number of high-quality research work in the field of experimental Physical Chemistry is still limited. In the present article I am going to give a brief overview on the high-quality work of some of the experimental Physical Chemist in India and their brief research topics are summarized below:
\end{abstract}

To write an article on Indian Experimental Physical Chemists a few names automatically come to our mind from the immediate previous generation, who made tremendous contributions in the experimental physical chemistry, are Professor Mihir Chowdhury, Professor Satya P Moulik and Professor J P Mittal. Professor Moulik contributed immensely on the thermodynamics, kinetics, electrochemistry, surface and colloid chemistry. Professor Chowdhury and Professor Mittal are the pioneers in laser chemistry in India. Both of them immensely contributed in fast kinetics and reaction dynamics through laser spectroscopy. In addition to that Professor Mittal developed pulse-radiolysis technique for the first time in India to monitor the reaction kinetics/dynamics by exciting the samples with electron beam to follow electron and proton transfer reaction.

The former director of IACS Kolkata (presently visiting professor IISER Bhopal) Professor Kankan Bhattacharyya has tremendous contribution in the physical chemistry. His research interest include confocal microscope, Fluorescence Correlation Spectroscopy (FCS) and fluorescence life time imaging, femtosecond up-conversion, live cell, drug delivery, proteins, fluorescent gold nano-clusters. He has been serving as a senior editor of one of the prestigious journal in the field of pure physical chemistry, the Journal of Physical Chemistry C (JPC C), over the last one decade. The chair professor from IISC Bangalore, Professor D D Sarma is well known for his contribution in several topics including experimental physical chemistry. His research theme incorporates strongly correlated electron systems, and nanocrystals in the quantum confinement regime. Presently he is serving as a senior editor of the prestigious ACS Energy Letters journal. Professor Tapas Chakraborty, IACS Kolkata is another scientist who is doing lot of good research in the physical chemistry. Laser-induced fluorescence and infrared spectra of cold molecules and molecular complexes, neutral as well as ions, under supersonic jet expansion and also in inert gas matrixes are main thrust of his research. Professor Haridas Pal from BARC Mumbai has a lot of contribution in the photochemistry, supramolecular chemistry and physical chemistry. $\mathrm{He}$ contributes a lot in the research of Marcus electron transfer in different system using fluorescence based steady-state and time-resolved (nanosecond to femtosecond) techniques. The research group of Professor S Sampath, IISC Bangalore is working on surfaces, interfaces and materials electrochemistry. The institute chair professor, Professor T Pradeep from IIT Madras is doing very high-quality research in several topics including experimental physical chemistry during the last three decades. He is serving as an Associate editor of ACS Sustainable Chemistry $\&$ Engineering journal. Professor George Thomas at IISER TVM has made significant contributions in several areas of photosciences and nanomaterials

*Author for Correspondence: E-mail:hnghosh@inst.ac.in,hnghosh2004@gmail.com 
which include design and study of photoresponsive nano materials, photosensitizers and fullerene-based donor-acceptor systems. Professor Ashok Ganguli from IIT Delhi (former Director of INST Mohali) has a good contribution to the fundamental understanding of different physical processes in several nanomaterials. The contribution to the research work on polymer chemistry by Professor Arun Nandi (IACS Kolkata) and Professor S Ramakrishnan also needs to be acknowledged. Professor S Ramakrishnan is also serving as an associate editor of the journal Chem. Comm. Professor Arun Chattopadhyay, from IIT Guwahati is doing good research in material science and its physical chemistry. His research topics involves thin films, membrane sciences, and nano science and technologies. The research of Professor Amitava Patra from IACS Kolkata (presently Director, INST Mohali) includes the understanding of the physical process such as energy transfer, and electron transfer of QD, $\mathrm{Au}$ nanoparticles based luminescent nanoparticles. Professor Narayan Pradhan of IACS Kolkata involves in the research of the physical process of the optical materials (QD and perovskites) and the doped nanocrystals. He has been named as top 5 author worldwide in JPC letters in the last two years. Professor T P Radhakrishnan (Univ. of Hyderabad) has done quite good contributions in the molecular materials. The research work on experimental biological physics and bio-nano interface by Professor Samir Kumar Pal at S N Bose, Kolkata needs to be acknowledged. Professor Nilmoni Sarkar from IIT Kharagpur has lot of contribution in the experimental physical chemistry in terms of photochemistry and spectroscopy. He has been named as top 5 author worldwide in JPCB in the last year. Another top 5 author in JPCB in the last year, Professor G B Dutt from BARC Mumbai also has very good contribution in photochemistry of molecules.

The present director of IISER Bhopal, Professor Siva Umapathy (earlier at IISC) is engaged in the research of Raman and other ultrafast laser spectroscopy of several probes (both molecules and materials). He is serving as an associate editor of the journal Spectrochimica Acta. Professor PK Das from IISC Bangalore also working on nonlinear optics, chemical dynamics and biophysical chemistry.Two former professors from TIFR Mumbai, Professor N Periasamy and Professor G Krishnamoorthy, has appreciable contribution in the fundamental understanding of physical processes in different materials using fluorescence spectroscopy. Another physical chemist, Professor Anunay Samanta at University of Hyderabad is working on ultrafast spectroscopy. His present research includes fundamentals of photo-induced processes of nanomaterials. The previous acting director of INSTMohali, Professor Hirendra N Ghosh (earlier who was in BARC, Mumbai) is doing very high-quality research in pure experimental physical chemistry who has been named as top 5 author in JPCC in the last two consecutive years (2016 and 2017). His present research interest includes ultrafast spectroscopy of newly synthesized nanomaterials (both plasmonic and semiconductor quantum dots) for energy related application. Professor Anindya Datta in IIT Bombay is involving in the research on excited state dynamics of different probe molecules using ultrafast spectroscopy. The research work by Professor Sudipta Maiti at TIFR Mumbai on biophotonics and bio-physics on different protein aggregates using multiphoton UV microscopy, fiber FCS has very good contribution. Professor Sanjay Wategaonkar, also at TIFR Mumbai, did quite a good research work on the gas phase spectroscopy to understand the weak interactions namely van der Waals and hydrogen bonding interactions. The contribution by Professor Ranjit Biswas at S N Bose, Kolkata on time resolved electronic spectroscopy needs to be appreciated.

Recently, a few young scientists from different part of India is doing very good research in the physical chemistry. Dr. Angshuman Nag at IISER Pune is doing high-quality physical chemistry research on colloidal nanocrystals, its photophysics and optoelectronics. Dr. Pankaj Mondal (also at IISER Pune) is working on the fundamental physical chemistry of nanomaterials using time resolved terahertz spectroscopy. Dr. Sameer Sapra, from IIT Delhi is working on the synthesis and energy related applications of nanocrystals. Understanding the noncovalent interactions present in the biomolecules as well as materials are the major thrust in the research group of Dr. Aloke Das at IISER Pune. Dr. Saptarshi Mukherjee from IISER Bhopal is also working on high-quality research on ultrafast and molecular spectroscopy. Dr. Pratik Sen from IIT Kanpur and Dr. Sobhan Sen at JNU are doing good research on the fundamental physical chemistry using ultrafast 
spectroscopy as well. Dr. Prasun K Mandal at IISER Kolkata is working on single molecule spectroscopy, ultrafast spectroscopy and microscopy of several fluorescent probes. The recent research work by Dr. Partha Hazra at IISER Pune on lyotropic liquid crystalline materials is also appreciable. Dr. Mahesh Hariharan (IISER Thiruvananthapuram) is pursuing high quality research on artificial photosynthesis using femtosecond spectroscopy. Dr. Samrat Mukhopadhyay at IISER Mohali is doing quality research on intrinsically disordered proteins and the study of single-molecule, ultrafast and nanoscale biophysics. The recent research work on spectroscopy of nanomaterials from the research group of Dr. Anshu Pandey, IISC Bangalore is also appreciable. The research on ultrafast reaction mechanisms and novel photomaterials by the group of Dr. Jyotishman Dasgupta, TIFR Mumbai is also worth being noted.

High-quality research on experimental physical chemistry demands researchers to develop certain good techniques which need both good funding and skill. The contribution of Professor Kankan Bhattacharya on the first development of ultrafast fluorescence spectroscopy during 90's in India needs to be acknowledged in this regard. The contribution of Professor Siva Umapathy at IISC Bangalore (presently at IISER Bhopal) is appreciable for the first-time development of different time resolved Raman spectroscopic techniques in India. Professor Dipak Palit and Professor H N Ghosh (who recently developed ultrafast laboratory at INST Mohali) jointly developed the ultrafast transient absorption (TA) spectroscopy at BARC for the first-time in India during late 90's. Later, several Indian physical chemists developed their own ultrafast laser laboratory: Professor Anindya Datta (IIT Bombay, Ultrafast TA and Upconversion spectroscopy), Professor Anunay Samanta (Univ. of Hyderabad, TA and Upconversion spectroscopy), Professor Amitava Patra (IACS, Upconversion), Dr. Anshu Pandey (IISC Bangalore, TA), Dr. Jyotishman Dasgupta (TIFR, TA and FSRS), Dr. Pankaj Mondal (IISER Pune, THz Spectroscopy), Dr. Prasun K Mandal (IISER Kolkata, TA, single molecule spectroscopy, microscopy) etc. 